\title{
Diploid genome differentiation conferred by RNA sequencing-based survey of genome-wide polymorphisms throughout homoeologous loci in Triticum and Aegilops
}

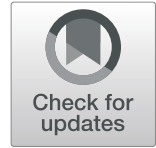

\author{
Sayaka Tanaka ${ }^{1}$, Kentaro Yoshida ${ }^{1 *}$ (D) Kazuhiro Sato ${ }^{2}$ (D) and Shigeo Takumi ${ }^{1}$ (i)
}

\begin{abstract}
Background: Triticum and Aegilops diploid species have morphological and genetic diversity and are crucial genetic resources for wheat breeding. According to the chromosomal pairing-affinity of these species, their genome nomenclatures have been defined. However, evaluations of genome differentiation based on genomewide nucleotide variations are still limited, especially in the three genomes of the genus Aegilops: Ae. caudata L. (CC genome), Ae. comosa Sibth. et Sm. (MM genome), and Ae. uniaristata Vis. (NN genome). To reveal the genome differentiation of these diploid species, we first performed RNA-seq-based polymorphic analyses for C, M, and $\mathrm{N}$ genomes, and then expanded the analysis to include the 12 diploid species of Triticum and Aegilops.

Results: Genetic divergence of the exon regions throughout the entire chromosomes in the $\mathrm{M}$ and $\mathrm{N}$ genomes was larger than that between $A$ - and $A^{m}$-genomes. Ae. caudata had the second highest genetic diversity following Ae. speltoides, the putative $B$ genome donor of common wheat. In the phylogenetic trees derived from the nuclear and chloroplast genome-wide polymorphism data, the $C, D, M, N, U$, and $S$ genome species were connected with short internal branches, suggesting that these diploid species emerged during a relatively short period in the evolutionary process. The highly consistent nuclear and chloroplast phylogenetic topologies indicated that nuclear and chloroplast genomes of the diploid Triticum and Aegilops species coevolved after their diversification into each genome, accounting for most of the genome differentiation among the diploid species.
\end{abstract}

Conclusions: RNA-sequencing-based analyses successfully evaluated genome differentiation among the diploid Triticum and Aegilops species and supported the chromosome-pairing-based genome nomenclature system, except for the position of Ae. speltoides. Phylogenomic and epigenetic analyses of intergenic and centromeric regions could be essential for clarifying the mechanisms behind this inconsistency.

Keywords: Genome-wide polymorphisms, Genome differentiation, RNA sequencing, Wheat

\footnotetext{
* Correspondence: kentaro.yoshida@port.kobe-u.ac.jp

'Graduate School of Agricultural Science, Kobe University, Rokkodai 1-1,

Nada-ku, Kobe 657-8501, Japan

Full list of author information is available at the end of the article
}

(c) The Author(s). 2020 Open Access This article is licensed under a Creative Commons Attribution 4.0 International License, which permits use, sharing, adaptation, distribution and reproduction in any medium or format, as long as you give appropriate credit to the original author(s) and the source, provide a link to the Creative Commons licence, and indicate if changes were made. The images or other third party material in this article are included in the article's Creative Commons licence, unless indicated otherwise in a credit line to the material. If material is not included in the article's Creative Commons licence and your intended use is not permitted by statutory regulation or exceeds the permitted use, you will need to obtain permission directly from the copyright holder. To view a copy of this licence, visit http://creativecommons.org/licenses/by/4.0/ The Creative Commons Public Domain Dedication waiver (http://creativecommons.org/publicdomain/zero/1.0/) applies to the data made available in this article, unless otherwise stated in a credit line to the data. 


\section{Background}

Crop domestication first occurred more than 10,000 years before the present. Since the early domestication process, ancient and modern breeders have utilized related wild species as genetic resources for crop improvement [1]. Recent and future climate change requires more efficient use of the useful genes in wild relatives [2, 3]. Elucidating the precise phylogenetic relationships among crops and their wild relatives will provide basic information for the use of agriculturally important genes found in the wild.

Genera Triticum and Aegilops include diverse diploid and allopolyploid species. The allopolyploid species are allotetraploids and allohexaploids, which were established through interspecific crossings between close and distinct relatives followed by chromosome doubling. In addition to allopolyploidization, nuclear differentiation at the diploid level drives speciation in this plant group. Genome differentiation was initially defined and updated based on the bivalent formation in meiotic cells of interspecific hybrids among related species in Triticum and Aegilops $[4,5]$. The homoeologous chromosomes of the diploid genomes are distinguished by in situ hybridization patterns of highly repetitive sequences and C-banding patterns, indicating that genome differentiation of diploid wheat and its relatives manifests at least partly in the distribution of heterochromatin and the accumulation of highly repetitive sequences [6]. Certain repetitive sequences such as retrotransposons rapidly and dramatically increase in their copy numbers in evolutionary-specific lineages [7-9], implying that repetitive sequence-based approaches would not necessarily reflect genetic relationships among related species. The use of genome-wide exon sequences, therefore, should be considered for clarifying the evolutionary relationships among related genomes.

Comprehensive studies on organellar genome diversity among Triticum and Aegilops using alloplasmic lines of common wheat have revealed diverse effects of differentiated chloroplast and mitochondrial genomes on various phenotypic and physiological traits [10-13]. The phylogenetic tree of organellar genomes is based on the maternal parents of Triticum and Aegilops allopolyploids and phylogenetic relationships among the organellar genomes of diploid species. Mitochondrial genomes have diverged in parallel with the chloroplast genomes of Triticum and Aegilops [12, 13]. Organellar DNA variations are significantly correlated with phenotypes in alloplasmic wheat lines [12]. Studies based on chloroplast nucleotide sequences have also clarified the phylogenetic relationships among chloroplast genomes in the tribe Triticeae, including the diploid Triticum and Aegilops species $[14,15]$. According to these previous reports, the phylogenetic relationship of the organellar genomes among Triticum and Aegilops is inconsistent with the one based on chromosome-pairing affinity. The position of Aegilops speltoides Tausch, an organellar genome donor of tetraploid and hexaploid wheat species, is especially discordant between the chromosome-pairingbased and organellar genome-based methods.

RNA sequencing (RNA-seq) has been a useful approach to survey genome-wide polymorphisms, including singlenucleotide polymorphisms (SNPs) and insertions/deletions (indels), in several wheat diploid relatives [16-23]. RNA-seq-derived polymorphism information is readily available to develop PCR-based markers such as cleaved amplified polymorphic sequences (CAPS) in target chromosomal regions. In this study, we conducted RNAseq analyses for three diploid Aegilops species, namely Ae. caudata L. (syn. Ae. markgrafii Hammer, CC genome), Ae. uniaristata Vis. (NN genome), and Ae. comosa Sibth. et $\mathrm{Sm}$. (MM genome). The three species are useful genetic resources for introgression of disease resistance into common wheat $[24,25]$. Aegilops caudata accessions are distributed from Greece to the northern part of Iraq [26]. Ae. uniaristata and Ae. comosa belong to the section Comopyrum, and have limited distribution in northwestern Turkey and from northwestern Turkey to Greece, respectively [27]. Comopyrum species are utilized for identifying novel alleles of glutenin subunit genes [28, 29]. Despite their usefulness as genetic resources, little genome information has been accumulated from these three Aegilops species.

The research objectives of the present study were (1) to survey RNA-seq-based polymorphisms through all chromosomes in the $\mathrm{C}, \mathrm{M}$, and $\mathrm{N}$ genome diploid species, (2) to convert the polymorphisms into genome-specific PCR-based markers, and (3) to clarify the phylogenetic relationships among the diploid Triticum and Aegilops species using exon-derived genome-wide polymorphism data.

\section{Results \\ Genome-wide genetic variations in three diploid Aegilops species}

To clarify the nucleotide variations in Ae. caudate (CC genome), Ae. uniaristata (NN genome), and Ae. comosa (MM genome), RNA-seq for a total of 15 accessions of these species was performed (Additional file 1: Fig. S1 and Table S1), generating 4,530,173 to 6,296,846 paired reads for each accession. After filtering out low-quality reads, 3,007,539 to 5,040,664 read pairs were obtained for the subsequent analyses (Additional file 1: Table S2). Of the filtered reads, 66.86 to $97.24 \%$ were aligned to $A$ e. tauschii genome sequences (Additional file 1: Table S3). Alignment rate variations were detected between the accessions of each species, and the alignment rate was not dependent on species. SNP and indel calling based on the short read alignments identified 13,401 to 135,902 SNPs and 177 to 1646 indels between Ae. caudata and 
Ae. tauschii, 14,880 to 86,171 SNPs and 220 to 1528 indels between Ae. comosa and Ae. tauschii, and 20,901 to 184,593 SNPs and 278 to 2273 indels between Ae. uniaristata and Ae. tauschii (Additional file 1: Table S3). These SNPs and indels covered all the chromosomes of Ae. tauschii (Additional file 1: Fig. S2). Of these SNPs, $83,018,61,704$, and 106,652 sites were polymorphic in Ae. caudate, Ae. comosa, and Ae. uniaristata, respectively (Additional file 1: Table S4). The distributions of the polymorphic sites over the chromosomes were not strikingly different among the three species (Fig. 1a and Additional file 1: Table S4).

\section{Development of $\mathrm{M}$ and $\mathrm{N}$ genome-specific markers and their utility}

To develop $\mathrm{M}$ and $\mathrm{N}$ genome-specific makers, we identified 13,600 fixed SNPs between Ae. comosa (MM genome) and Ae. uniaristata (NN genome) that can discriminate $\mathrm{M}$ and $\mathrm{N}$ genomes. A fixed SNP site is monomorphic within a species, while it has different nucleotides between species. These fixed SNPs between Ae. comosa and Ae. uniaristata covered all the chromosomes (Fig. 1b). Each chromosome had 1729 to 2249 fixed SNPs (Additional file 1: Table S5). When compared to the number of fixed SNPs between Ae. comosa and Ae. caudata and between Ae. uniaristata and Ae. caudata, the number of fixed SNPs between Ae. comosa and Ae. uniaristata was small. This result is consistent with the taxonomic classification: these two species belong to the same section Comopyrum. Three CAPS markers were designed based on these fixed SNPs (Additional file S1: Fig. S3 and Table S6). These CAPS markers successfully discriminated $\mathrm{N}$ and $\mathrm{M}$ genomes.
Phylogenetic relationships among diploid Triticum and Aegilops species based on SNPs in the coding regions of nuclear genomes

To reveal the phylogenetic relationships of diploid Triticum and Aegilops species, we utilized the previously published RNA-seq data of Ae. tauschii (DD genome) [19], Ae. umbellulata (UU genome) [20], einkorn wheat (AA and $\mathrm{A}^{\mathrm{m}} \mathrm{A}^{\mathrm{m}}$ genomes) [23], and Stiopsis species (SS genome) [21], combining it with our current data from Ae. caudata (CC genome), Ae. comosa (MM genome), and Ae. uniaristata (NN genome) (Additional file 1: Table S7). The qualified $300 \mathrm{bp}$ paired-end short reads of all the species were aligned to the Ae. tauschii genome sequences (Additional file 1: Table S8), generating a set of 109,980 non-redundant SNPs (Additional file 1: Table S9). Considering that the non-redundant SNPs were distributed over all the chromosomes (Fig. 2), SNPs could be regarded as representative SNPs that adequately reflect the nuclear genome evolution of the diploid Aegilops/Triticum species. Another set of 108,618 non-redundant SNPs for the diploid Aegilops/Triticum species, including Hordeum vulgare as an outgroup species, was prepared for the phylogenetic analyses (Fig. 2 and Additional file 1: Table S9). Due to the lower alignment rate of $H$. vulgare to RNA-seq reads of the Ae. tauschii reference genome (Additional file 1: Table S8), the number of non-redundant SNPs within the diploid Triticum and Aegilops species was reduced when $H$. vulgare was included (Additional file 1: Table S9).

Phylogenetic trees of the diploid Triticum and Aegilops species were constructed using neighbor-joining (NJ) and maximum likelihood (ML) methods (Fig. 3). All the phylogenetic trees with/without outgroup species $H$.

\section{A}

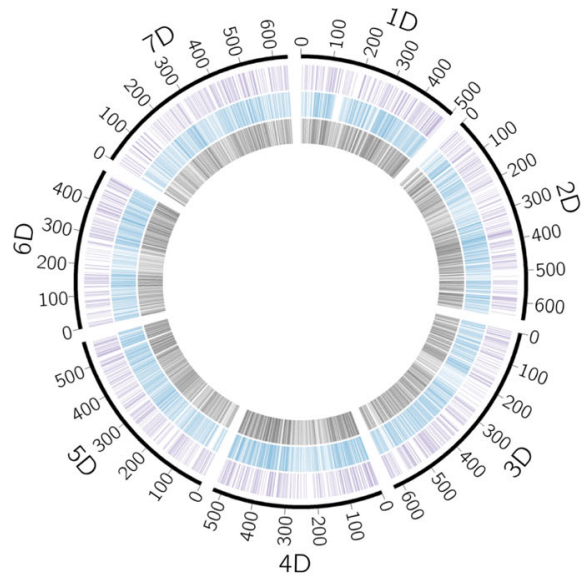

B

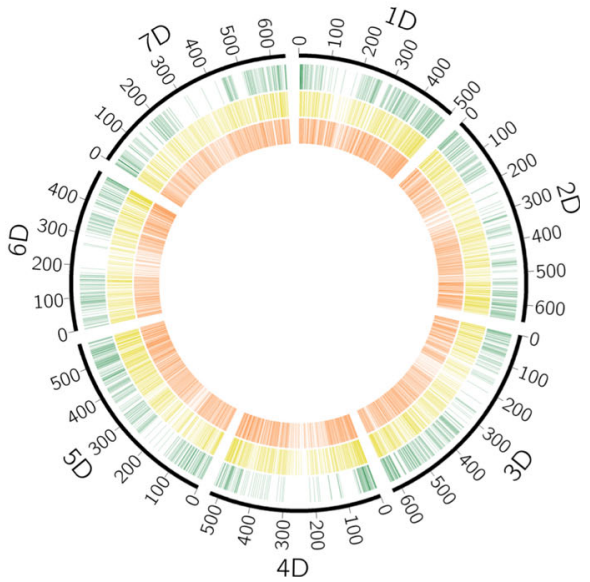

Fig. 1 Distribution of polymorphic sites and fixed SNPs within/between Aegilops caudata (CC genome), Ae. comosa (MM genome), and Ae. uniaristata (NN genome). a The CIRCOS plot visualizes polymorphic sites within species. Violet, blue, and black lines indicate polymorphic sites within Ae. uniaristata, Ae. Comosa, and Ae. caudata, respectively. b Green, yellow, and orange lines indicate fixed SNPs between Ae. comosa and Ae. uniaristata, between Ae. caudata and Ae. comosa, and between Ae. caudata and Ae. uniaristata, respectively 

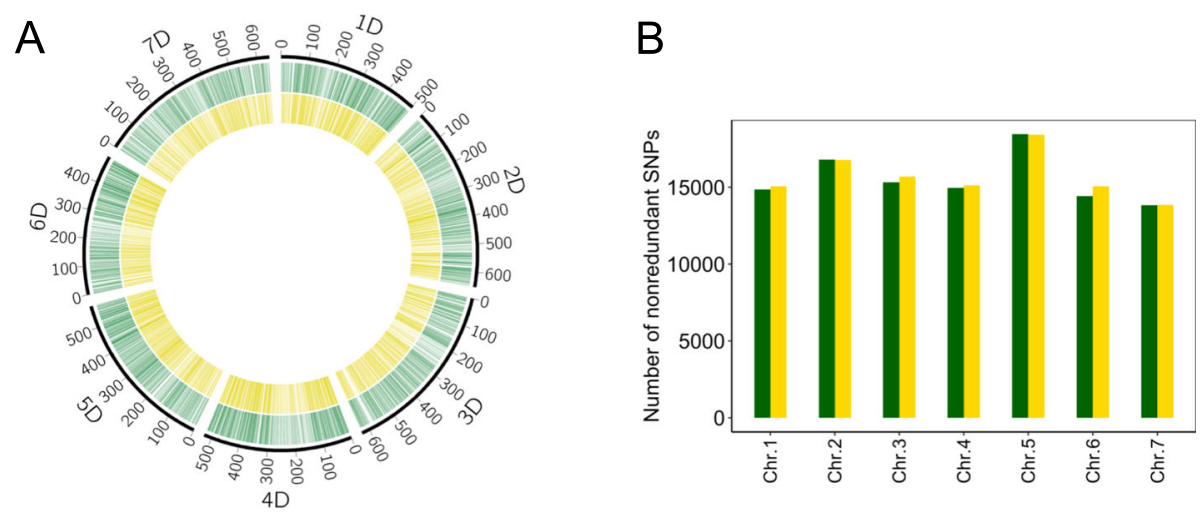

Fig. 2 Distribution of non-redundant SNPs over the chromosomes of nuclear genomes. Distributions of non-redundant SNPs with/without outgroup species are visualized by a CIRCOS plot (a). Green and yellow lines represent positions of non-redundant SNPs with and without outgroups species over the chromosomes, respectively. The number of non-redundant SNPs for each chromosome is shown as a barplot (b). Green and yellow bars indicate non-redundant SNPs with and without outgroup species, respectively

vulgare showed the same topology, which was consistent with the topology of the previously reported phylogenetic trees based on RNA-seq [22]. The diploid species having the same genome were classified into the same clades with $100 \%$ bootstrap probability, except for Sitopsis species. Section Sitopsis was separated into two clades that correspond to the subsections Emaginata and Truncata [21, 22]. Subsection Emaginata was more closely related to D-genome species. As reported by Glémin et al. 2019 [22], Triticum and Aegilops species are classified into three large clades: einkorn wheat (A and $\mathrm{A}^{\mathrm{m}}$ genomes), Truncata ( $\mathrm{S}$ genomes), and other species $(C, D, M, N, U$, and $S$ genomes that were further classified into $S^{\mathrm{s}} S^{\mathrm{s}}, S^{\mathrm{l}} S^{\mathrm{l}}$, and $S^{\mathrm{b}} S^{\mathrm{b}}$ ). As expected, $\mathrm{M}$ and $\mathrm{N}$ genome species belonging to the section Comopyrum had the closest relationship. $\mathrm{C}$ genome species were more closely related to $\mathrm{U}$ genome species than to $\mathrm{M}$ and $\mathrm{N}$ genome species. The branch length between $\mathrm{M}$ and $\mathrm{N}$ genome species was longer than that between $\mathrm{A}$ and $\mathrm{A}^{\mathrm{m}}$ genome species, and was slightly smaller than that between $\mathrm{C}$ and $\mathrm{U}$ genome species.

Since the phylogenetic tree confirmed the genome differentiation between the diploid species, we investigated the distribution of unique nucleotide substitutions over the chromosomes that discriminated between each of the genomes (Fig. 4 and Additional file S1: Fig. S4). When non-redundant SNPs were monomorphic within a species and distinct from the other diploid species of Aegilops and Triticum, they were regarded as unique nucleotide substitutions. In this analysis, the $\mathrm{S}$ genome species of the section Emaginata were assembled into one group. In every genome, unique nucleotide substitutions covered all chromosomes with some differences in their density.
Nucleotide polymorphisms within each nuclear genome To evaluate the level of nucleotide polymorphisms for diploid Triticum and Aegilops species, we used the number of pairwise nucleotide differences between accessions within species as an indicator of genetic diversity (dissimilarity), which was calculated based on the set of non-redundant SNPs excluding $H$. vulgare. The usage of non-redundant SNPs without missing values enables us to compare genetic diversity among species on an equal basis. Genetic diversity was quite distinct among the diploid Triticum and Aegilops species (Fig. 5). Following Ae. speltoides, Ae. caudata had the second highest genetic diversity among the diploid Triticum and Aegilops species. In Ae. caudata, Ae. tauschii, and T. monococcum ssp. aegilopoides (Link) Thell. (syn. T. boeoticum Boiss), the number of pairwise nucleotide differences depended on the pairs of accessions, implying the existence of genetically divergent groups within their species. This observation is consistent with previous reports of Ae. tauschii and T. monococcum ssp. aegilopoides indicating that these two species contain more than two divergent groups $[19,23,30]$. T. urartu, T. monococcum ssp. monococcum, and Ae. searsii showed lower genetic diversity than the other diploid Triticum and Aegilops species.

\section{Phylogenetic relationships of the organelle genomes of diploid Triticum and Aegilops species}

RNA-seq short reads of the diploid Triticum and Aegilops species were aligned to the chloroplast genome of Ae. tauschii. The alignment rate of short reads was dependent on the accessions (Additional file 1: Table S3 and Table S8), and the alignment rate for some accessions was over $30 \%$. This high percentage could be due to a large amount of chloroplast RNA contained in the 


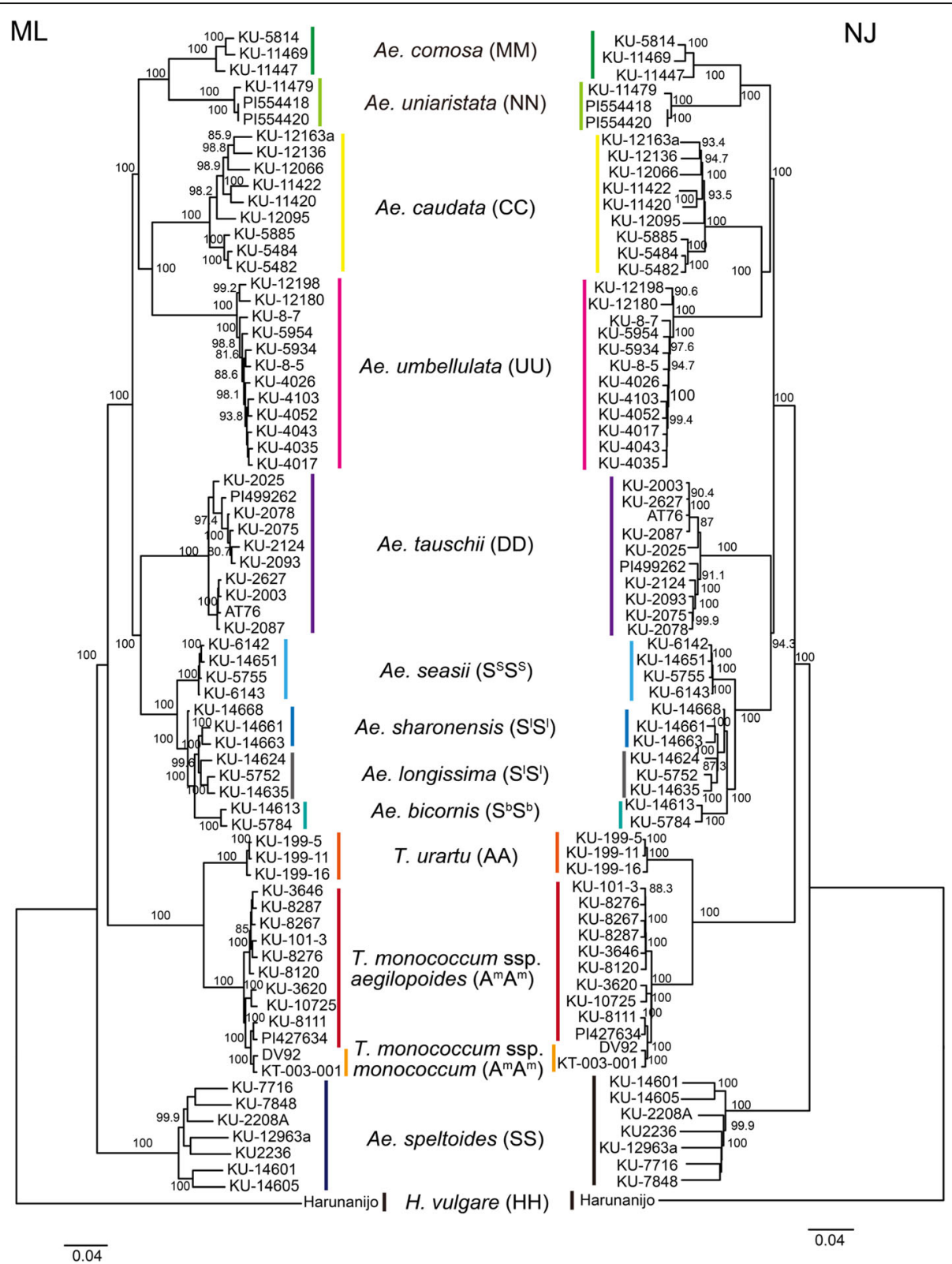

Fig. 3 Phylogenetic relationship among diploid Triticum and Aegilops species. A maximum-likelihood tree and a neighbor-joining tree are shown. The trees were constructed based on 108,618 non-redundant SNPs in the nuclear genome. The number next to each branch indicates bootstrap probability based on 1000 replications

sampled leaves from these accessions and/or could result from misalignment of RNA-seq short reads that should be mapped to the nuclear genome. After detecting SNPs for each accession and combining them, we obtained 234 non-redundant SNPs in the chloroplast genome. In order to address organelle genome evolution, a phylogenetic tree was constructed based on these non-redundant SNPs using the ML method (Fig. 6). The topology of the phylogenetic tree was highly consistent with that based on SNPs of the nuclear genome, but the following minor differences existed in the topology. In the chloroplast genome, after separation from the einkorn wheat (AA and $\mathrm{A}^{\mathrm{m}} \mathrm{A}^{\mathrm{m}}$ genomes) and Ae. speltoides (SS genome), Ae. tauschii (DD genome) first diverged from the other Aegilops species. Also, Ae. caudata (CC genome) showed a non-monophyletic pattern. Three accessions of Ae. caudata were more closely related to Ae. umbellulata (UU genome), while the other accessions of Ae. caudata were close to Ae. comosa (MM genome) and Ae. uniaristata (NN genome). In the nuclear trees, $\mathrm{S}$ genome species for subsection Emaginata and D, C, M, N, and U genome species formed a monophyletic clade, indicating that they 


\begin{tabular}{|c|c|c|}
\hline Genome & Chr. 1D & Chr. 2D \\
\hline M & 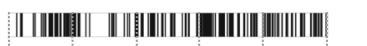 & || ||||||||||||||||||||||||||||||||||||||||||||||| \\
\hline $\mathrm{N}$ & |||||||||||| & |||||||||||||||||||||| $\mid$ \\
\hline c & ||||||||||||||||||||||| ||||||||||||||||||||||||||| & $\||||||||||| \mid$ \\
\hline D & ||||||||||| |||||||||||||||||||||||||||| & 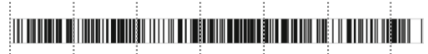 \\
\hline S (Truncata) & 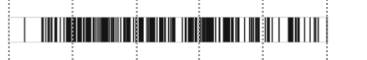 & | |||||||||||||||||||||||||||||||||||||||||||||||||| \\
\hline$S$ (Emaginata) & 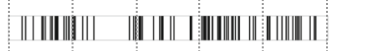 & 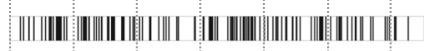 \\
\hline A & ||||||||||||||||||| - | - || & |||||||||||||||||| \\
\hline$A^{m}$ & 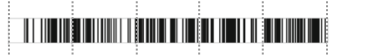 & 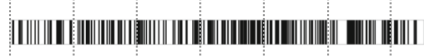 \\
\hline U & |||||||||||||||||||||||||||||||||| & ||||||||||||||||||||||||||||||||||||||||||||||||||||| \\
\hline & $\begin{array}{lllll}0 & 100 & 200 & 300 & 40\end{array}$ & $0 \quad 100 \quad 200 \quad 300 \quad 400$ \\
\hline
\end{tabular}

Fig. 4 Distribution of unique SNPs that discriminated between genomes over each chromosome. The unique SNPs for each genome were mapped to the chromosomes of Ae. tauschii. Black bars indicate SNP positions. The figure shows the distribution of the unique SNPs on chromosomes 1D and 2D. The results for other chromosomes are shown in Additional file S1: Fig. S4

diverged from one common ancestor, and Ae. caudata was a monophyletic group.

\section{Discussion}

Clear differentiation between Ae. comosa and Ae. uniaristata despite their phenotypic similarity

Our RNA-seq-based phylogenetic analyses using SNPs in nuclear and chloroplast genomes showed that Ae. uniaristata and Ae. comosa, belonging to the section Comopyrum, were the most closely related species among the diploid Triticum and Aegilops species. Both species belonged to a monophyletic clade, suggesting

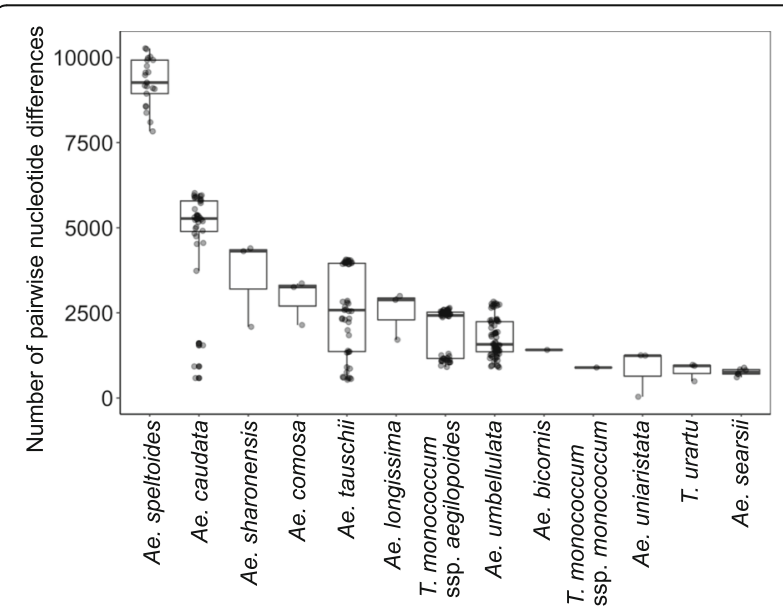

Fig. 5 Distinct genetic diversity among diploid Triticum and Aegilops species. A boxplot with jitter points representing the number of nucleotide difference between individual accessions within species is shown. Each translucent grey point indicates one pairwise comparison between two accessions. Darker points indicate overlaps of points. The median of each species in the boxplot clarifies distinct genetic diversity between species and jitter points disclose discontinuities in nucleotide differences between accessions within species that they originated from one common ancestor. This observation is consistent with the nuclear and chloroplast phylogenetic relationships of published studies that have used different sets of accessions and the different methods for detecting nucleotide variations $[15,22]$.

Our study indicates high genetic divergence between Ae. uniaristata and Ae. comosa, which was higher than that between $\mathrm{A}$ and $\mathrm{A}^{\mathrm{m}}$ genomes (Fig. 3), even though the morphologies of Ae. uniaristata and Ae. comosa are similar. Unique nucleotide substitutions that discriminate them from other genomes were distributed over the chromosomes in both species (Fig. 4 and Additional file S1: Fig. S4). Considering that coding regions are generally more conservative than intergenic regions, which are mostly composed of repetitive sequences and transposable elements, the intergenic regions are expected to have higher genetic divergence. In fact, there are distinct in situ hybridization patterns of highly repetitive sequences and C-banding patterns between $\mathrm{M}$ and $\mathrm{N}$ genomes [6]. Nucleotide differences between both species may thus cause non-preferential chromosome pairing between $\mathrm{M}$ and $\mathrm{N}$ genomes [31]. Whole genome sequence comparisons, including intergenic regions, will be necessary for understanding the relationship between genome differentiation and chromosome-pairing affinity.

Genome differentiation in nuclear and chloroplast genomes in diploid Triticum and Aegilops species

The observed short internal branches in the phylogenetic trees of nuclear and chloroplast genomes suggest that Triticum and Aegilops species emerged during a relatively short period in the past and then the nuclear and chloroplast genomes each diverged (Fig. 6). For the nuclear genome, first, the $\mathrm{S}$ genome of the section Truncata was separated from the other genomes, and then 


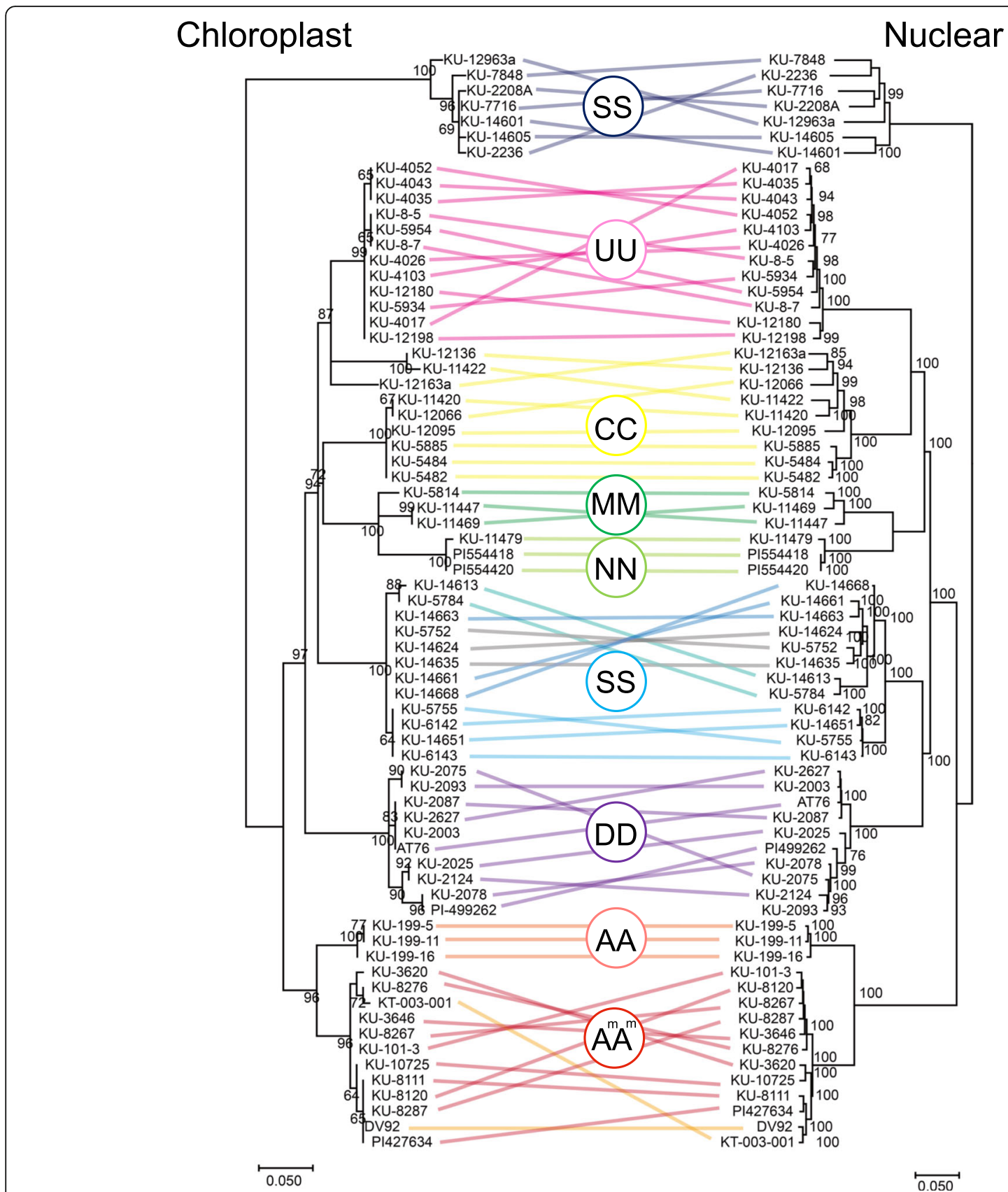

Fig. 6 Genome differentiation of chloroplasts and nuclei of diploid Triticum and Aegilops species. Maximum likelihood phylogenetic trees based on 234 non-redundant SNPs of chloroplasts and nuclei are shown. The same accessions in the trees are connected with colored lines. Different colors are used for each species. Letters in the colored circles represent genomes. Bootstrap probabilities based on 1000 replications are shown next to the branches

the $\mathrm{A}$ and $\mathrm{A}^{\mathrm{m}}$ genomes of einkorn species were separated from a common ancestor of $\mathrm{S}, \mathrm{C}, \mathrm{D}, \mathrm{M}, \mathrm{N}$, and $\mathrm{U}$ genomes (Figs. 3and 6). S, D, M, N, and U genomes form a monophyletic clade. Their common ancestor diverged into two groups: one is composed of $U, C, M$, and $\mathrm{N}$ genomes, and the other is of $\mathrm{S}$ and $\mathrm{D}$ genomes. This observation is consistent with a previously proposed scenario of the evolutionary history of Aegilops/Triticum species [22]. In contrast, for the chloroplast genome, after separating from $\mathrm{A}$ and $\mathrm{A}^{\mathrm{m}}$ genomes, the $\mathrm{D}$ genome diverged from the $\mathrm{C}, \mathrm{D}, \mathrm{M}, \mathrm{N}$, and $\mathrm{S}$ genomes. The $\mathrm{C}$ genome species exhibited a polyphyletic relationship. Considering that these minor inconsistencies between the nuclear and chloroplast genomes were 
observed only in the short internal branches of the phylogenetic tree (Fig. 6), genome differentiation of the chloroplast might have occurred independently of that of the nucleus during the short emergence period of $\mathrm{S}$, $\mathrm{D}, \mathrm{M}, \mathrm{N}$, and $\mathrm{U}$ genomes. On the other hand, considering the close consistency of the phylogenetic relationships between the nuclear and chloroplast genomes, both genomes could have coevolved after diversification of each genome.

The phylogenetic relationship of the organellar genomes of Triticum and Aegilops is inconsistent with their chromosome-pairing affinity [12-15]. In particular, the chromosome-pairing-based and organellar genome-based relationships conflict at the position of Ae. speltoides. The current and previous phylogenetic analyses using RNA-seq based SNPs indicate that Ae. speltoides (SS genome) belonging to the section Truncata is separate from the other SS genome species belonging to the section Emaginata $[21,22]$. There are apparent discrepancies in the relationships of $\mathrm{S}$ genomes between the exon sequences of their nuclear genomes and chromosome-pairing affinity. Intergenic regions, including transposable elements and centromeric regions, occupy most of the genomes. Meiotic associations of centromeres precede chromosome arm pairing. After initial associations of nonhomologous centromeres, homologous centromere pairing is considered to be established by assessments of DNA sequence homology, although the mechanisms behind centromere paring are not fully understood [32]. The study of fission yeast implies that noncoding RNA is involved in homologous chromosome pairing [33]. Supportive results of heterochromaticmediated paring have been found in Caenorhabditis elegans, Drosophila, and Arabidopsis [32]. Intergenic regions and epigenetic mechanisms potentially contribute to the chromosome-pairing affinity among Triticum and Aegilops species. Phylogenomic and epigenetic analyses of the intergenic regions may provide clues for solving the contradiction between the nuclear and chloroplast genomes and the chromosome-pairing affinity.

Some SNPs from chloroplast genomes are potentially derived from mutations by RNA editing [34]. These SNPs, however, should affect only the length of the external branches in the phylogenetic tree. Informative SNPs for the tree construction could be caused by nucleotide substitutions in the evolutionary history of Triticum/Aegilops species rather than mutations via RNA editing, because RNA editing occurs in mRNA from each chloroplast in each individual species. Nucleotide sequences of chloroplast genomes from RNA-seq represent average nucleotide sequences of mRNA from the chloroplast genome of an individual. Most SNPs produced via RNA editing can be detected as heterozygous SNPs. Our analysis used only homozygous SNPs and enabled us to reduce the effects of RNA editing on the topology of the phylogenetic trees.

\section{Nucleotide polymorphisms in diploid Triticum and Aegilops species}

Ae. speltoides had the highest nucleotide diversity among the tested diploid species. Only Ae. speltoides is an outcrossing species, while the others in our study are selfing species. The mating system of outcrossing increases effective population size and recombination events, both of which positively affect genome-wide genetic diversity [35]. Although RNA-seq can detect only SNPs in expressed genes, the detected SNPs covered all the chromosomes. The number of nucleotide differences between individuals could be regarded as a representative value of genome-wide genetic diversity.

Although it is possible to underestimate nucleotide diversity due to the small number of analyzed accessions in some species, our study revealed varying levels of genetic diversity among $T$. monococcum ssp. aegilopoides, Ae. caudata, Ae. umbellulata, and Ae. tauschii, although they are all selfing species (Fig. 5). Their effective population sizes, population structures, and demographic histories such as speed and time of colonization of their modern habitats could be the factors that generated distinct genetic diversity. After Ae. speltoides, which is an outcrossing species, Ae. caudata had the second highest nucleotide diversity. In the phylogenetic tree of the nuclear genome, Turkish and Iraqi accessions (KU-5482, KU-5484, KU-5885) and Greek accessions of Ae. caudata were genetically divergent (Fig. 3). Greek accessions had long external branches. If individuals of Ae. caudata are regarded as a representative of a local population, genetic differences between the Greek accessions could reflect isolation between local populations.

Genetic diversity in Ae. tauschii was higher than that in T. monococcum ssp. aegilopoides and Ae. umbellulata. Ae. tauschii has the broadest range of habitats from the Middle East to China. Three genetically divergent sublineages, TauL1, TauL2, and TauL3, are detected in Ae. tauschii [30]. Genetic differences between the sublineages explain the higher genetic diversity in $A e$. tauschii [19]. These sublineages have contrasting geographical distributions. TauL2 is mainly distributed in Transcaucasia and the Middle East. The TauL1 sublineage can be divided into TauL1a and TauL1b. The habitat of TauL1a is endemic to the Transcaucasus and the Middle East, while the habitat of TauL1b expands to East Asia. The phenological change to early flowering and the gain of $\mathrm{NaCl}$-induced-stress tolerance in TauL1b have contributed to its colonization of semi-arid Central Asian habitats [36, 37]. The higher genetic diversity could be linked to its successful colonization of diverse habitats, which may have accelerated genetic differentiation between the sublineages due to the limited gene flow between its remote habitats. 
The observed pattern of $T$. monococcum ssp. aegilopoides was caused by nucleotide divergence between two groups, one of which includes the ancestor of the diploid cultivar T. monococcum ssp. monococcum [23]. On the other hand, Ae. umbellulata had lower genetic diversity and a star-like phylogeny with short external branches, suggesting recent expansion into modern habitats [20]. Polyphyly of Ae. caudata has been reported in a previous study based on four regions of the chloroplast genome [14]. In this study, the chloroplast genomes of Ae. caudata also showed a polyphyletic pattern. One of the groups was closer to Ae. umbellulata, while the others were closer to Ae. comosa and Ae. uniaristata.

\section{Conclusions}

The RNA-seq approach uncovered genetic divergence of the exon regions through all of the chromosomes of $\mathrm{C}$, $\mathrm{M}$, and $\mathrm{N}$ genome diploid species, allowing us to construct novel CAPS makers that discriminated between $\mathrm{M}$ and $\mathrm{N}$ genomes. Phylogenetic trees based on the exon-derived genome-wide polymorphism data overall supported the differentiation of nuclear and chloroplast genomes of diploid Triticum and Aegilops species. Chromosomal pairing-affinity between $\mathrm{S}$ genomes, however, was contradicted by their genetic differentiation of exon regions. In order to resolve this inconsistency, phylogenomic and epigenetic analyses of intergenic regions and centromeric regions are needed in future studies.

\section{Methods}

\section{Plant materials}

Seeds of nine accessions of Ae. caudata, three accessions of Ae. uniaristata, and three accessions of Ae. comosa were supplied by the National BioResource Project (NBRP)-Wheat, Japan (https://www.nbrp.jp) for KUnumbered accessions and by the National Small Grains Research Facility, USDA-ARS, USA for PI-numbered accessions (Additional file 1: Table S1). These accessions were collected in Greece, Iraq, and Turkey.

\section{RNA sequencing}

Total RNA was extracted using Sepasol-RNA I Super G (Nacalai Tesque, Kyoto, Japan) from the mature leaves of 2-month-old plants in the field of Kobe University, Kobe, Japan. After DNase I treatment, libraries for 300-bp paired-end RNA-seq were constructed using 6 to $10 \mu \mathrm{g}$ of total RNA using the TruSeq RNA Library Preparation Kit v2 (Illumina, San Diego, CA, USA) according to a previously reported protocol [38]. Sequencing was conducted on an Illumina MiSeq sequencer. The obtained reads were deposited to the DDBJ Sequence Read Archive under accession number DRA009411. To evaluate genome differentiation between the diploid Triticum and Aegilops species, we also used published RNA-seq reads that have been generated using the same protocol and sequencing platform: 10 accessions of Ae. tauschii [19], 12 accessions of Ae. umbellulata [20], 19 accessions of the section Sitopsis, 15 accessions of the einkorn wheat [23], and one accession of $H$. vulgare [38] (Additional file 1: Table S7). These RNA-seq reads were obtained from the DDBJ Sequence Read Archives.

\section{Quality control, alignment of RNA-seq reads, and molecular evolutionary analyses}

Quality control was conducted according to Miki et al. [21]. Adapter sequences and low-quality bases with an average quality score per 4 bp of $<30$ were removed, and reads of fewer than $50 \mathrm{bp}$ were filtered out using Trimmomatic software, version 0.33 [39]. The qualified paired reads were aligned to the reference genome sequence of Ae. tauschii [40], and the chloroplast genomes (NCBI reference sequence: NC_022133.1) were aligned using HISAT software, version 2.1.0 [41]. The same procedure as described by Nishijima et al. (2016) [19] was used to detect SNPs and indels. SNPs and indels were called using Coval [42] under the following criteria: the depth of read coverage was $\geq 10$, and $>95 \%$ of the aligned reads designated different nucleotides from the referenced ones. We chose positions of SNPs at which no ambiguous nucleotides were detected and the read depth was $\geq 10$, generating a set of nonredundant SNPs for nuclear, chloroplast, and mitochondrial genomes of the tested species. Based on nonredundant SNPs, fixed SNPs that distinguished $\mathrm{C}, \mathrm{M}$, and $\mathrm{N}$ genomes were detected. CIRCOS, version 0.33 [43] was used for visualization of $\mathrm{SNP} /$ indel distributions over the chromosomes of Ae. tauschii. Phylogenetic trees were constructed based on the set of nonredundant SNPs. MEGA, version 10 [44] was used for the construction of maximum likelihood (ML) trees and neighbor-joining trees (NJ).

\section{Marker development}

CAPS markers were designed based on the fixed SNPs between $\mathrm{M}$ - and $\mathrm{N}$ - genomes. Primer sets were generated with Primer3plus software [45] (Additional data 1: Table S6). The candidate SNPs were converted to CAPS markers. PCR amplification, digestion of the PCR products, and visualization of their digested fragments were conducted as described in our previous study [46].

\section{Supplementary information}

Supplementary information accompanies this paper at https://doi.org/10. 1186/s12864-020-6664-3.

Additional file 1: Table S1. Accessions of C, M, and $N$ genome species used for RNA-seq analyses. Table S2. Quality control of RNA-seq reads of C, M, N-genome diploid wheat accessions. Table S3. Alignment rate and 
SNPs and indels for $\mathrm{C}, \mathrm{M}$, and $\mathrm{N}$ genome species. Table S4. Polymorphic sites in Ae. caudata (CC genome), Ae. comosa (MM genome), and Ae. uniaristata (NN genome). Table S5. Fixed SNPs between Ae. caudata (CC genome), Ae. comosa (MM genome), and Ae. uniaristata (NN genome). Table S6 List of CAPS markers to distinguish M and $\mathrm{N}$ genomes. Table S7 Plant materials used for phylogenetic and polymorphic analyses. Table S8 Alignment rate and SNPs and indels for diploid Triticum and Aegilops species used for phylogenetic and polymorphic analyses. Table S9 Sets of non-redundant SNPs in diploid Triticum and Aegilops species estimated using RNA-seq. Figure $\mathbf{S 1}$ Geographic distribution of diploid Triticum and Aegilops species that were analyzed in this study. Figure S2 Distribution of SNPs and indels of Ae. caudata, Ae. comosa, and Ae. uniaristata. In the CIRCOS visualizations, green, yellow, and orange indicate $A$ e. uniaristata, Ae. comosa, and Ae. caudata, respectively. SNPs (A) and indels (B) for Ae. uniaristata KU-11479, PI554418, and PI554420; Ae. comosa KU5814, KU-11447, and KU-11469; and Ae. caudata KU-5482, KU-5484, KU5885, KU-11420, KU-11422, KU-12066, KU-12095, KU-12136, and KU12136a are shown from the outer to the inner circles. Figure S3 CAPS markers that discriminate $\mathrm{M}$ or $\mathrm{N}$ genomes from $\mathrm{A}$ and $\mathrm{B}$ genomes. Figure S4 Distribution of unique SNPs that discriminate other genomes over each chromosome. The unique SNPs for each genome were mapped to the chromosomes of Ae. tauschii. Black bars indicate SNP positions. The figure shows the distribution of the unique SNPs on the chromosomes $3 D, 4 D, 5 D, 6 D$, and $7 D$.

\section{Abbreviations}

RNA-seq: RNA-sequencing; SNPs: Single nucleotide polymorphisms; CAPS: Cleaved amplified polymorphic sequences; PCR: Polymerase chain reaction; ML: Maximum likelihood; NJ: Neighbor-joining

\section{Acknowledgments}

Seeds of the diploid wheat species used in this study were supplied by NBRP-Wheat, Japan, and USDA-ARS, USA. Computations for RNA sequence assembly of reads were performed on the NIG supercomputer at the ROIS National Institute of Genetics, Japan.

\section{Authors' contributions}

Designed the experiments and the analyses: ST, KY, KS, ST. Performed the experiments: ST, KS. Analyzed the data: ST, KY. Wrote the paper: ST, KY, ST. All authors read and approved the final manuscript.

\section{Funding}

The design of the study, molecular evolutionary analyses, interpretation of data and writing the manuscript were supported by Grant-in-Aid for Scientific Research (B) No. 16H04862 from the Ministry of Education, Culture, Sports, Science and Technology (MEXT) of Japan to ST, by Grant-in-Aid for Scientific Research on Innovative Areas No. $19 H 04863$ from MEXT to ST. The sequencing and transcriptome analyses were supported by MEXT as part of a Joint Research Program implemented at the Institute of Plant Science and Resources, Okayama University, Japan. The transcriptome analyses, interpretation of data and writing the manuscript were supported by PRESTO (No. JPMJPR15QB) from JST to KY.

\section{Availability of data and materials}

All data generated or analyzed during this study are included in this published article in Additional file 1 and DDBJ Sequence Read Archive under accession number DRA009411. The sets of unique SNPs for each species that were used for Fig. 4 and Additional file S1: Fig. S4 are available at https:// github.com/PlantGeneticsKobeU/Diploid-Aegilops-species-unique-SNPs.

\section{Ethics approval and consent to participate}

Not applicable.

\section{Consent for publication}

Not applicable

\section{Competing interests}

The authors declare that they have no competing interests.

\section{Author details}

Graduate School of Agricultural Science, Kobe University, Rokkodai 1-1, Nada-ku, Kobe 657-8501, Japan. ${ }^{2}$ Institute of Plant Science and Resources, Okayama University, Chuo 2-20-1, Kurashiki 710-0046, Japan.

\section{Received: 27 December 2019 Accepted: 10 March 2020}

Published online: 20 March 2020

\section{References}

1. Janzen GM, Li W, Hufford MB. The extent of adaptive wild introgression in crops. New Phytol. 2019;221(3):1279-88.

2. Moore G. Strategic pre-breeding for wheat improvement. Nat Plants. 2015;1:15018.

3. Prohens J, Gramazio P, Plazas M, Dempewolf H, Kilian B, Díez MJ, Fita A, Herraiz FJ, Rodríguez-Burruezo A, Soler S, Knapp S, Vilanova S. Introgressiomics: a new approach for using crop wild relatives in breeding for adaptation to climate change. Euphytica. 2017;213:158.

4. Kihara H. Genomanalyse bei Triticum und Aegilops. IX. Systematischer Aufbau der Gattung Aegilops auf genomanalytischer Grundlage. Cytologia (Tokyo). 1949;14:135-44.

5. Lilienfeld FA. Kihara: genome-analysis in Triticum and Aegilops X. Concluding review. Cytologia (Tokyo). 1951;16:101-23.

6. Badaeva ED, Friebe B, Gill BS. Genome differentiation in Aegilops. 1. Distribution of highly repetitive DNA sequences on chromosomes of diploid species. Genome. 1996;39(2):293-306.

7. Tsukahara S, Kobayashi A, Kawabe A, Mathieu O, Miura A, Kakutani T. Bursts of retrotransposition reproduced in Arabidopsis. Nature. 2009;461(7262):423-6.

8. Tsukahara S, Kawabe A, Kobayashi A, Ito T, Aizu T, Shin-I T, Toyoda A, Fujiyama A, Tarutani Y, Kakutani T. Centromere-targeted de novo integrations of an LTR retrotransposon of Arabidopsis lyrata. Genes Dev. 2012;26(7):705-13.

9. Zhang QJ, Gao LZ. Rapid and recent evolution of LTR retrotransposons derives rice genome evolution during the speciation of AA-genome Oryza species. G3. 2017;7(6):1875-85.

10. Tsunewaki K, Wang GZ, Matsuoka Y. Plasmon analysis of Triticum (wheat) and Aegilops. 1. Production of alloplasmic common wheats and their fertilities. Genes Genet Syst. 1996;71(5):293-311.

11. Tsunewaki K, Wang GZ, Matsuoka Y. Plasmon analysis of Triticum (wheat) and Aegilops. 2. Characterization and classification of 47 plasmons based on their effects on common wheat phenotype. Genes Genet Syst. 2002;77(6): 409-27.

12. Wang GZ, Miyashita N, Tsunewaki K. Plasmon analyses of Triticum (wheat) and Aegilops: PCR-single-strand conformational polymorphism (PCR-SSCP) analyses of organellar DNAs. Proc Natl Acad Sci U S A. 1997;94(26):14570-7.

13. Wang GZ, Matsuoka Y, Tsunewaki K. Evolutionary features of chondriome divergence in Triticum (wheat) and Aegilops shown by RFLP analysis of mitochondrial DNAs. Theor Appl Genet. 2000;100(2):221-31.

14. Yamane K, Kawahara T. Intra- and interspecific phylogenetic relationships among diploid Triticum-Aegilops species (Poaceae) based on base-pair substitutions, indels, and microsatellites in chloroplast noncoding sequences. Am J Bot. 2005;92(11):1887-98.

15. Bernhardt N, Brassac J, Kilian B, Blattner F. Dated tribe-wide whole chloroplast genome phylogeny indicates recurrent hybridizations within Triticeae. BMC Evol Biol. 2017;17:141.

16. lehisa JCM, Shimizu A, Sato K, Nasuda S, Takumi S. Discovery of highconfidence single nucleotide polymorphisms from large-scale de novo analysis of leaf transcripts of Aegilops tauschii, a wild wheat progenitor. DNA Res. 2012;19(6):487-97.

17. lehisa JCM, Shimizu A, Sato K, Nishijima R, Sakaguchi K, Matsuda R, Nasuda S, Takumi S. Genome-wide marker development for the wheat D genome based on single nucleotide polymorphisms identified from transcripts in the wild wheat progenitor Aegilops tauschii. Theor Appl Genet. 2014;127(2):261-71.

18. Fox SE, Geniza M, Hanumappa M, Naithani S, Sullivan C, Preece J, Tiwari VK, Elser J, Leonard JM, Sage A, Gresham C, Kerhornou A, Bolser D, MCCarthy F, Kersey P, Lazo GR, Jaiswal P. De novo transcriptome assembly and analyses of gene expression during photomorphogenesis in diploid wheat Triticum monococcum. PLoS One. 2014;9(5):e96855.

19. Nishijima R, Yoshida K, Motoi Y, Sato K, Takumi S. Genome-wide identification of novel genetic markers from RNA sequencing assembly of diverse Aegilops tauschii accessions. Mol Gen Genomics. 2016;291(4):1681-94. 
20. Okada M, Yoshida K, Nishijima R, Michikawa A, Sato K, Takumi S. RNA-seq analysis reveals considerable genetic diversity and provides genetic markers saturating all chromosomes in the diploid wild wheat relative Aegilops umbellulata. BMC Plant Biol. 2018:18(1):271.

21. Miki Y, Yoshida K, Mizuno N, Nasuda S, Sato K, Takumi S. Origin of wheat Bgenome chromosomes inferred from RNA sequencing analysis of leaf transcripts from section Sitopsis species of Aegilops. DNA Res. 2019;26(2): 171-82.

22. Glémin S, Scornavacca C, Dainat J, Burgarella C, Viader V, Ardisson M, Sarah G, Santoni S, David J, Ranwez V. Pervasive hybridizations in the history of wheat relatives. Sci Adv. 2019;5(5):eaav9188.

23. Michikawa A, Yoshida K, Okada M, Sato K, Takumi S. Genome-wide polymorphisms from RNA sequencing assembly of leaf transcripts facilitate phylogenetic analysis and molecular marker development in wild einkorn wheat. Mol Gen Genomics. 2019:294(5):1327-41.

24. Valkoun J, Hammer K, Kucerova D, Bartos P. Disease resistance in the genus Aegilops L. -stem lust, leaf rust, stripe rust, and powdery mildew. Kulturpflanzen. 1985;33:133-53.

25. Riar AK, Kaur S, Dhaliwal HS, Singh K, Chhuneja P. Introgression of a leaf rust resistance gene from Aegilops caudata to bred wheat. J Genet. 2012;91: 155-61.

26. Ohta S. Genetic differentiation and post-glacial establishment of the geographical distribution in Aegilops caudata L. Gene Genet Syst. 2000;75:189-96.

27. Maxted N, White K, Valkoun J, Konopka J, Hargreaves S. Towards a conservation strategy for Aegilops species. Plant Genet Resour. 2008;6:126-41.

28. Wang K, Gao L, Wang S, Zhang Y, Li Y, Zhang M, Xie Z, Yan Y, Belgard M, Ma W. Phylogenetic relationship of a new class of LMW-GS genes in the $m$ genome of Aegilops comosa. Theor Appl Genet. 2011;122(7):1411-25.

29. Tang H, Du X, Wang H, Ma X, Bo C, Li A, Kong L. Cloning and characterization of a novel low-molecular-weight glutenin subunit gene with an unusual molecular structure of Aegilops uniaristata. J Genet. 2018; 97(5):1463-8.

30. Mizuno N, Yamasaki M, Matsuoka Y, Kawahara T, Takumi S. Population structure of wild wheat D-genome progenitor Aegilops tauschii Coss.: implications for intraspecific lineage diversification and evolution of common wheat. Mol Ecol. 2010;19(5):999-1013.

31. Kimber $G$, Pignore D, Sallee PJ. The relationships of the $M$ and $M^{u}$ genomes of Triticum. Can J Genet Cytol. 1983:25(5):509-12.

32. Da Ines $\mathrm{O}$, White $\mathrm{Cl}$. Centromere associations in meiotic chromosome pairing. Annu Rev Genet. 2015;49:95-114.

33. Ding DQ, Okamasa K, Yamane M, Tsutsumi C, Haraguchi T, Yamamoto M, Hiraoka Y. Meiosis-specific noncoding RNA mediates robust pairing of homologous chromosomes in meiosis. Science. 2012;336(6082):732-6.

34. Shikanai T. RNA editing in plant organelles: machinery, physiological function and evolution. Cell Mol Life Sci. 2006;63(6):698-708.

35. Ellegren $H$, Galtier N. Determinants of genetic diversity. Nat Rev Genet. 2016;17(7):422-33.

36. Matsuoka Y, Takumi S, Kawahara T. Intraspecific lineage divergence and its association with reproductive trait change during species range expansion in central Eurasian wild wheat Aegilops tauschii Coss. (Poaceae). BMC Evol Biol. 2015:15:213

37. Saisho D, Takumi S, Matsuoka Y. Salt tolerance during germination and seedling growth of wild wheat Aegilops tauschii and its impact on the species range expansion. Sci Rep. 2016;6:38554.

38. Sato K, Tanaka T, Shigenobu S, Motoi Y, Wu J, Itoh T. Improvement of barley genome annotations by deciphering the Haruna Nijo genome. DNA Res. 2016:23(1):21-8

39. Bolger AM, Lohse M, Usadel B. Trimmomatic: a flexible trimmer for Illumina sequence data. Bioinformatics. 2014:30(15):2114-20.

40. Luo MC, Gu YQ, Puiu D, Wang H, Twardziok SO, Deal KR, Huo N, Zhu T, Wang L, Wang Y, McGuire PE, Liu S, Long H, Ramasamy RK, Rodriguez JC, Van SL YL, Wang Z, Xia Z, Xiao L, Anderson OD, Ouyang S, Liang Y, Zimin AV, Pertea G, Qi P, Bennetzen JL, Dai X, Dawson MW, Müller HG, Kugler K, Rivarola-Duarte L, Spannagl M, KFX M, Lu FH, Bevan MW, Leroy P, Li P, You FM, Sun Q, Liu Z, Lyons E, Wicker T, Salzberg SL, Devos KM, Dvořák J. Genome sequence of the progenitor of the wheat $D$ genome Aegilops tauschii. Nature. 2017;551(7681):498-502.

41. Kim D, Langmead B, Salzberg SL. HISAT: a fast spliced aligner with low memory requirements. Nat Methods. 2015;12(4):357-60.
42. Kosugi S, Natsume S, Yoshida K, MacLean D, Cano L, Kamoun S, Terauchi R. Coval: improving alignment quality and variant calling accuracy for nextgeneration sequencing data. PLoS One. 2013;8(10):e75402.

43. Krzywinski M, Schein J, Birol I, Connors J, Gascoyne R, Horsman D, Jones SJ, Marra MA. Circos: an information aesthetic for comparative genetics. Genome Res. 2009;19(9):1639-45.

44. Kumar S, Stecher G, Li M, Knyaz C, Tamura K. MEGA X: Molecular evolutionary genetics analysis across computing platforms. Mol Biol Evol. 2018:35(6):1547-9.

45. Untergasser A, Nijveen $H$, Rao X, Bisseling T, Geurts R, Leunissen JA. Primer3Plus, an enhanced web interface to Primer3. Nucleic Acids Res. 2007;35:W71-4.

46. Sakaguchi K, Nishijima R, lehisa JCM, Takumi S. Fine mapping and genetic association analysis of Net2, the causative D-genome locus of low temperature-induced hybrid necrosis in interspecific crosses between tetraploid wheat and Aegilops tauschii. Genetica. 2016;144(5):523-33.

\section{Publisher's Note}

Springer Nature remains neutral with regard to jurisdictional claims in published maps and institutional affiliations.
Ready to submit your research? Choose BMC and benefit from:

- fast, convenient online submission

- thorough peer review by experienced researchers in your field

- rapid publication on acceptance

- support for research data, including large and complex data types

- gold Open Access which fosters wider collaboration and increased citations

- maximum visibility for your research: over $100 \mathrm{M}$ website views per year

At $\mathrm{BMC}$, research is always in progress.

Learn more biomedcentral.com/submissions 